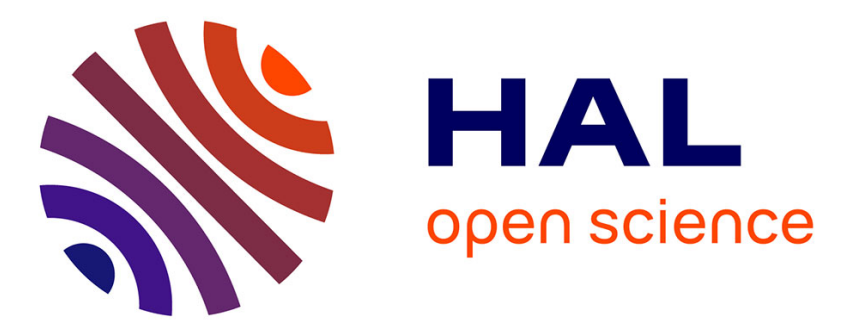

\title{
Dynamic analysis of wooden rockfall protection structures subjected to impact loading using a discrete element model
}

Ignacio Olmedo, Franck Bourrier, Frédéric Berger, Ali Limam, David Bertrand

\section{To cite this version:}

Ignacio Olmedo, Franck Bourrier, Frédéric Berger, Ali Limam, David Bertrand. Dynamic analysis of wooden rockfall protection structures subjected to impact loading using a discrete element model. European Journal of Environmental and Civil Engineering, 2020, 24 (9), pp.1430-1449. 10.1080/19648189.2018.1472042 . hal-01824003

\section{HAL Id: hal-01824003 \\ https://hal.science/hal-01824003}

Submitted on 8 Nov 2018

HAL is a multi-disciplinary open access archive for the deposit and dissemination of scientific research documents, whether they are published or not. The documents may come from teaching and research institutions in France or abroad, or from public or private research centers.
L'archive ouverte pluridisciplinaire HAL, est destinée au dépôt et à la diffusion de documents scientifiques de niveau recherche, publiés ou non, émanant des établissements d'enseignement et de recherche français ou étrangers, des laboratoires publics ou privés. 


\title{
Dynamic analysis of wooden rockfall protection structures subjected to impact loading using a Discrete Element model
}

\author{
I. Olmedo ${ }^{\mathrm{a}, \mathrm{b}}$ and F. Bourrier ${ }^{\mathrm{b}}$ and D. Bertrand ${ }^{\mathrm{c}}$ and F. Berger ${ }^{\mathrm{b}}$ and A. Limam ${ }^{\mathrm{c}}$ \\ ${ }^{a}$ GTS, 38420 Domène, France; b Irstea Grenoble, UR EMGR, 38402 St-Martin d'Hères, \\ France; ${ }^{\mathrm{C} I N S A-L y o n, ~} 69621$ Villeurbanne, France
}

\section{ARTICLE HISTORY}

Compiled November 8, 2018

\begin{abstract}
Wooden structures made of felled trees are used in forested slopes as protection structures against rockfall. A model based on the Discrete Element Method is developed to analyse their response to normal impacts of blocks. The interaction between the blocks and the stems and the stem response are explicitly integrated. After the model calibration using impact tests, a wide range of impact scenarii representative of real configurations is explored. The influence of three parameters (stem diameter, impact velocity and block diameter) is analysed.

Three impact types were identified for different ratios $R_{D}$ between the block and stem diameters. For small $R_{D}$, the impact is limited to a brief contact. For intermediate $R_{D}$, successive contacts of similar duration are observed. After the last contact, the block is sent back with a large velocity. For large $R_{D}$, two contacts are observed. The second contact can be assimilated to a quasi-static loading of the stem. In addition, structure damage increases for increasing $R_{D}$ ranging from almost no damage for the first impact type to rupture of the stem for the third type. The largest $R_{D}$ associated with the first impact type is the target configuration that favors block energy decrease and limits structure damage.
\end{abstract}

\section{KEYWORDS}

Rockfall ; Wooden structures ; Protection structures ; Fresh wood ; Impact ; Discrete Element Method

\section{Introduction}

In the field of civil engineering, numerous models have been developed to assess the efficacy of a wide range of protection structures against rockfall hazard: metallic fences (Bertrand, Trad, Limam, \& Silvani, 2012; Cazzani, Mongiovi, \& Frenez, 2002; Gottardi, \& Govoni, 2009; Volkwein, Roth, Gerber, \& Vogel, 2009) or embankments (Lambert, \& Bourrier, 2013; Plassiard, \& Donze, 2009; Ronco, Oggeri, \& Peila, 2009), for example. These numerical approaches are of interest as they allow accounting for the physics involved in the modeling of block impacts onto complex structures. Numerical simulations enable to analyse the structures and to propose recommendations for their design. However, experimental data remain necessary to calibrate and validate the numerical models. Given the technical difficulties and economic costs to carry out in-field real-scale impact experiments, reduced scale laboratory tests are an interesting 
alternative to get accurate experimental data.

In alpine forested slopes, specific wooden structures made of felled trees are increasingly installed as a complement to the protection provided by the forest stand itself (Dorren, Berger, Le Hir, Mermin, \& Tardif, 2005). Contrary to the impact of blocks on standing trees, (Dorren, Berger, \& Putters, 2006; Jonsson, 2007), the impact of blocks on these wooden protection structures has not been investigated.

Recent experimental laboratory studies enabled to characterize the dynamic response of small stems (up to $10 \mathrm{~cm}$ of diameter) to impacts (Bertrand, Bourrier, Olmedo, Brun, Berger, \& Limam, 2013; Olmedo, Bourrier, Bertrand, Toe, Berger, \& Limam, 2015) with the final aim of understanding the response of wooden protection structures to a block impact. A first Discrete Element Method (DEM) based model (Olmedo, Bourrier, Bertrand, Berger, \& Limam, 2016) of the stems response to impact loading was also proposed and allowed describing the dynamic response of the stems tested in the laboratory. In the simulations, the loading was imposed by the experimentally measured time evolution of the force at mid-span. Thus, the proposed model did not explicitly account for the block and its contact with the impacted system.

These studies focused on the dynamic response of fresh wood stems only. In a first step, the time evolution of the structure response, which is related with the degradation of the mechanical properties of the wood material along the stem composing the structure and in the vicinity of the structures boundaries, was not investigated. Consequently, these studies can only be used to analyze the response of a numerical model of protection devices made of felled trees considering their mechanical properties after setting up the structure.

The objective of this paper is to analyse the response of real scale wooden protection structures to the normal impact of a block after setting up the structure for design purposes. To meet this objective, the DEM model proposed and calibrated in Olmedo et al. (2016) has been improved by integrating a contact model describing the local interaction between the block and the fresh wood stem. After a calibration phase, the improved model was used to simulate real scale impacts on felled trees protection structures (tree diameters ranging from 35 to 80 centimeters) for a wide range of impact conditions. Numerical predictions were used to analyse the physical processes involved for different impact configurations and to identify the most favorable structure configurations in terms of block momentum reduction as well as structure damage and rupture. These observations were finally discussed to propose general recommendations for the design of these structures.

\section{Numerical modelling}

\subsection{Discrete Element model}

The DEM was used to model the mechanical response of a fresh stem to a dynamic loading (Olmedo et al., 2016). This method allows modeling the dynamic motion of particles that interact by contact or remote interactions (Cundall, \& Strack, 1979). At each time step, the particles' locations, orientations, geometries, and physical properties are used to calculate the sum of forces on each particle. Newton's law are then solved for all the particles, allowing the update of their positions and orientations. The DEM has been widely applied for the study of granular materials (O'Sullivan, 2012). It has also been used to model deformable structures (e.g. Bertrand et al., 2012; Effeindzourou et al., 2016). 
The numerical developments were implemented in the YADE-dem code (Smilauer, \& Chareyre, 2010). In the model, the fresh wood stems are considered homogeneous cylindrical bodies composed of nodes $(N)$ regularly located along the stem longitudinal axis $x$ (Figure 1). The nodes are linked by cylindrical connections $(C)$ (Bourrier, Kneib, Chareyre, \& Fourcaud, 2013) that can interact with external bodies, such as spheres. The model of the interactions between the nodes $(N)$, called stem model in the followings, governs the mechanical response of the deformable cylinder (i.e. a fresh wood stem). The model of the interactions of the cylindrical connections $(C)$ with external bodies, called contact model in the followings, controls the interaction between the cylindrical element (i.e. a fresh wood stem) and an external body (i.e. a block).

\subsubsection{Fresh wood stem model}

The stem model was initially developed and calibrated in Olmedo et al. (2016). It allows calculating the forces and moments between contiguous nodes depending on their relative positions and orientations, which determines the macroscopic response of the cylinder (i.e. stem). In the context of the study, the stems are mainly subjected to bending loads. Thus, the relation between node positions (resp. orientations) and interaction forces (resp. moments) associated with tensile, shear, or twist efforts are modeled using an elastic formulation that depends on the longitudinal modulus of elasticity (MOE) and the Poisson's ratio of the stem material.

A multi-linear relationship is used to model the response of the stem to bending loadings. The moments between contiguous nodes associated with bending loadings are the moments $M_{y}$ and $M_{z}$ (called $M$ in the following) along the $y$ and $z$ axes of the local coordinate system associated with the first node of the two contiguous ones. These moments are calculated incrementally depending on the nodes relative orientations $\theta_{y}$ and $\theta_{z}$ (called $\theta$ ) along the $y$ and $z$ axes (Figure 1b) and applied to both of the contiguous nodes.

A bi-linear relation describes the $M(\theta)$ loading phase (i.e. for increasing $\theta$ )(Figure $2)$. An elastic threshold moment $\left(M_{\text {Elast }}\right)$ allows defining the transition from the first linear relation, defined by the stiffness coefficient $k_{\text {Elast }}$, to the second one, associated with the coefficient $k_{N L}$. The unloading phase (i.e. for decreasing $\theta$ ) is also governed by a linear relation described by the coefficient $k_{U n l d}$.

Bourrier et al. (2013) and Olmedo et al. (2016) proposed relationships that relate the stiffness coefficients $k_{\text {Elast }}, k_{N L}$, and $k_{U n l d}$ to the stem cross section geometry, distance between the contiguous nodes, and material properties:

$$
\begin{gathered}
k_{\text {Elast }}=\frac{M O E I}{l} \\
k_{N L}=\frac{E_{N L} I}{l} \\
k_{\text {Unld }}=\frac{E_{U n l d} I}{l}
\end{gathered}
$$

where $M O E, E_{N L}$ and $E_{U n l d}$ are the longitudinal modulus of elasticity, the mod- 
ulus governing the second linear phase, and the modulus for the unloading phase, respectively. $l$ and $I$ are the distance between contiguous nodes and the area moment of inertia $\left(I=\pi D^{4} / 64\right)$, respectively. $D$ is the stem diameter at the node location.

When the bending moment $M_{\text {Rupt }}$ is reached, which corresponds to stem's failure, the interaction is considered broken and the simulation is stopped.

The elastic threshold $\left(\sigma_{\text {Elast }}\right)$ and the ultimate stress before failure $\left(\sigma_{\text {Rupt }}\right)$ are calculated from the geometrical properties, $M_{\text {Elast }}$, and $M_{\text {Rupt }}$ such as:

$$
\begin{gathered}
\sigma_{\text {Elast }}=\frac{M_{\text {Elast }}}{I} \frac{D}{2} \\
\sigma_{\text {Rupt }}=\frac{M_{\text {Rupt }}}{I} \frac{D}{2}
\end{gathered}
$$

$\sigma_{\text {Rupt }}$ assumption, even if not valid when material non-linearities develop, is widely accepted for the mechanical characterization of wood (Bergman, Cai, \& Carll, 2010). Indeed, it corresponds to the definition of the modulus of rupture $(M O R)$.

A viscous damping of the bending moment $M$ was finally implemented following an incremental formalism:

$$
d M_{\text {Damp }}=\beta k_{\text {Elast }} d \dot{\theta}
$$

where the increment of the damping contribution to bending $\left(d M_{D a m p}\right)$ is calculated from the increment of the relative angular velocity of the nodes $(d \dot{\theta})$ and a damping coefficient $(\beta)$.

Finally, the calibration parameters of the stem model are: $M O E, E_{N L}, E_{U n l d}, \sigma_{\text {Elast }}$, $\sigma_{\text {Rupt }}$, and $\beta$.

\subsubsection{Block - stem contact model}

The block-stem contact model added to the initial stem model consists in modelling the interactions between the cylindrical elements and external bodies. In the DEM framework, the contact model between two particles usually relates the contact forces to the interpenetration and to the relative velocities at the contact point. The contact plane, tangent to the contact surface at the contact point, enables to define the normal and tangential components of the contact force. In the context of the study, only the normal component is considered because impacts are assumed orthogonal to the cylinder longitudinal axis.

Previous results from Olmedo et al. (2016) showed that including a simple linear contact model was sufficient to significantly improve the relevance of the impact model. However, in this study, we choose to use a contact model integrating energy dissipation at the contact which allowed us to analyse energy dissipation processes during impact.

The normal contact force $(F)$ depends on the indentation $(\alpha)$ of the impactor into the cylinder (Figure 3). From the numerical point of view, it is described by the overlap between the block and the cylindrical element. In the absence of additional information concerning the highly variable block shape, a formulation corresponding to the Hertz-Crook contact theory for spheres developing an elasto-plastic response 
was chosen (Abrate, 1998; Crook, 1952). During the loading phase, the contact force can be expressed as:

$$
F^{\text {Load }}=k_{c} \cdot \alpha^{3 / 2}
$$

where $k_{c}$ is the contact stiffness, which can be calculated from the physical and geometrical properties of the colliding bodies (bodies 1 and 2):

$$
k_{c}=\frac{4}{3} E_{R} \sqrt{R}
$$

where,

$$
\begin{gathered}
\frac{1}{R}=\frac{1}{R_{1}}+\frac{1}{R_{2}} \\
\frac{1}{E_{R}}=\frac{1-\nu_{1}^{2}}{E_{R 1}}+\frac{1-\nu_{2}^{2}}{E_{R 2}}
\end{gathered}
$$

$R_{j}, E_{R j}$ and $\nu_{j}$ are the radius, radial modulus of elasticity, and Poisson's ratio of the body $j$.

For the unloading phase, the formulation proposed by Crook (Abrate, 1998; Crook, 1952), assuming negligibles permanent indentations, was implemented:

$$
F^{U n l d}=F_{m}\left(\frac{\alpha}{\alpha_{m}}\right)^{5 / 2}
$$

where $F_{m}$ is the maximum force reached during the loading phase and $\alpha_{m}$ is the corresponding indentation.

Finally, for this study, the calibration parameters of the contact model are $E_{R}$, and $\nu$ of each colliding body.

\subsection{Model calibration}

Olmedo et al. (2016) proposed a calibration of the stem model where the contact model was not taken into account, i.e. the loading was modelled by a time varying force measured experimentally. In this paper, a new calibration is proposed that accounts for the contact model to describe the interaction between the block and the stem.

\subsubsection{Impact experiments}

The chosen species for the tests was European beech (Fagus sylvatica) for being representative of the French Alps forests exposed to rockfall (i.e. European beech is present in the $25 \%$ of the french protection forests) (IGN, 2014). The wood pieces were extracted from the same forest patch at the Col de Vence forest (Rhone Alpes, France). Tested trees had similar growing conditions and were cut at the same period of the 
year to reduce mechanical properties variations. Stems were tested maximum one day after their cut to limit changes in wood moisture content.

The laboratory experiments on fresh wood stems consisted in impacting simply supported stems at mid-span with a Mouton-Charpy pendulum. Stems of diameters between 3 and 9 centimeters and of $1.5 \mathrm{~m}$ length between supports were impacted. Force cells (Siscam MC-100) enabled to measure the evolution in time of the impact force and of the reaction forces at the supports. A high speed camera (Photron Fastcam, Nikkor 35-80 lens) was installed to measure the impactor and stem's mid-span displacements. Each stem was impacted twice, at two different impact energy levels. Additional details on the experimental set-up can be found in Olmedo et al. (2015).

\subsubsection{Calibration procedure}

The model calibration was based on experimental results from Olmedo et al. (2015). The stems were modeled by deformable cylinders and the time evolution of the measured impact force was imposed at mid-span. The calibration was done by adjusting the calibration parameters to achieve a good fit between experimental and numerical impact work calculations $\left(W_{E x t}\right)$. The external work is defined such as:

$$
W_{E x t}=\int_{0}^{t} F d y_{b}
$$

where $F$ is the force applied onto the stem and $y_{b}$ is the impactor displacement at the instant $t$ (Olmedo et al., 2016). Thirty-seven impact tests were calibrated, which allowed to estimate the mechanical properties of the tested wood.

The calibration focused on the adjustment of the parameters concerning the stem $\left(M O E, E_{N L}, E_{U n l d}, \sigma_{\text {Elast }}, \sigma_{\text {Rupt }}, \beta\right)$.

For the pendulum, classical values for steel were used as parameters for the contact model : $E_{R 1}=210 G P a$ and $\nu=0.3$. For the stem, the parameter $E_{R 2}$ of the contact model was set at $160 \mathrm{MPa}$, as proposed by Quetel (2005), and the Poisson's ratio $\nu$ was set at 0.3 (Olmedo et al., 2016).

The experimental data from Olmedo et al. (2015) allowed adjusting the parameters of the stem model. The simulations consisted in impacting at mid-span a simply supported stem, represented by a cylinder, with a spherical discrete element, representing the pendulum. The measured impact velocity of the pendulum was imposed to the sphere at the beginning of the simulation. The parameters values obtained from the previous calibration of the stem model (Olmedo et al., 2016) were initially used. The calibration consisted in slight adjustments of some of these parameters to obtain the best agreement with experimental results. The numerical simulations were adjusted to fit the experimental results in terms of energy, $W_{E x t}$.

\subsection{Impact scenarii}

\subsubsection{Description}

After the calibration phase, the model was used to simulate blocks impacts on felled trees made structures corresponding to real scenarii in terms of field impact conditions. The analysis focused on the impact response of a simplified structure made of one tree. The structure was modeled as a deformable cylinder with simply supported boundary 
conditions (Figure 4), which is representative of real set-ups. Impacting blocks were considered as rigid spherical bodies with a translational velocity perpendicular to the longitudinal axis of the tree, and the block angular velocity was considered nil. Structures were impacted at mid-span and impacts were centered on tree's longitudinal axis.

For the design of structures, engineers and practitioners consider the characteristics of the expected rockfall events (in terms of block mass and velocities) to propose an $a d-h o c$ rockfall protection device capable to resist to the incoming impact energy. In particular, stem diameter needs to be defined for the setup of the structure.

Simulations were performed for several impact scenarii, characterized by the block diameter $\left(D_{b}\right)$ and impact velocity $\left(v_{i n}\right)$. Moreover, different stem diameters $\left(D_{t}\right)$ were considered. Thus, $D_{t}, D_{b}$ and $v_{i n}$ were considered as input variables. The stem length $L$ was set at $10 \mathrm{~m}$ for all simulations.

Concerning the impacting block, $D_{b}$ and $v_{i n}$ ranges are representative of rockfall events Volkwein et al. (2011)(Tab.1). Tree diameters $\left(D_{t}\right)$ ranges are representative of the current felled trees made protection devices installed in the European Alps (Tab.1). When $\frac{D_{b}}{D_{t}}>4$, it is assumed that the stem cannot absorb a significant amount of block energy (Dorren et al., 2005). The sets of parameters presenting $\frac{D_{b}}{D_{t}}>4$ were thus not considered. 1386 scenarii, corresponding to different values of $D_{b}, D_{t}$, and $v_{i n}$, were finally considered for the simulations.

\subsubsection{Mechanical properties of fresh wood for in-field scale simulations}

The fresh wood properties depend on many factors and the characteristics of each forest stand cannot be directly related to the experimental data presented in this paper. Despite this large variability, the fresh wood properties were assumed constant for all simulations. Given the very reduced amount of data in the literature concerning the mechanical properties of fresh wood at the tree scale, the choice was made to set up these mechanical properties depending on the results obtained during the model calibration.

The parameters $E_{R}$ and $\nu$ describing the contact model were set at the same values as in the calibration phase. The block's and stem's densities $\left(\rho_{b}\right.$ and $\left.\rho_{t}\right)$ were set at $2800 \mathrm{~kg} \cdot \mathrm{m}^{-3}$ and $1200 \mathrm{~kg} \cdot \mathrm{m}^{-3}$, respectively.

For the stem mechanical properties, the previous model calibration (Olmedo et al., 2016) provided a characterization of fresh wood mechanical properties for small diameter trees $\left(D_{t}<10 \mathrm{~cm}\right)$. Given the complexity of the tree's growing mechanisms, the mechanical properties of wood evolve along the tree (Keith., 1964) and radially (Lundstrom et al., 2007). In addition, the irregularities associated with growing conditions, branch insertions, or scars lead to significant variations on the mechanical properties of wood (Lundstrom et al., 2008). Consequently, the previous model calibration for small diameter trees $\left(D_{t}<10 \mathrm{~cm}\right)$ cannot be directly extrapolated to larger diameter trees $\left(D_{t}>35 \mathrm{~cm}\right)$.

The values obtained have been compared to previous studies found in the literature concerning large diameter stems ranging between $20 \mathrm{~cm}$ and $50 \mathrm{~cm}$. Lavers, \& Gwendoline (1974) proposed values of MOE and modulus of rupture $\left(M O R=\sigma_{R u p t}=\right.$ $\frac{32 M_{\text {Rupt }}}{\pi D^{3}}$ ) (Bodig and Jayne, 1982)) for fresh European beech stems with a $50 \%$ moisture content. They estimated the MOE at $9790 \mathrm{MPa}$ and the MOR at $64.8 \mathrm{MPa}$ from quasi-static bending tests. The differences between the previous model calibration values (Olmedo et al., 2016) and Lavers, \& Gwendoline (1974) estimations are not significant in view of the variability of wood properties (Bergman et al., 2010). The 
MOE measured by Lavers, \& Gwendoline (1974) is 9.6\% larger than the calibration value. The MOR measured by Lavers, \& Gwendoline (1974) is $10.3 \%$ lower. Provided that the values from the calibration described in section 2.2.2 do not differ substantially from these obtained in Olmedo et al. (2016), this comparative analysis will lead to use the values obtained from the calibration of the model that explicitly account for block-stem contact for the simulations for in-field structures made of large diameter trees.

\subsubsection{Energy transfers}

The main goal of the design of a rockfall protection device is to maximize the block's energy loss due to the impact. In addition, the study of the energy transfers and dissipations on the protection structure is mandatory to develop optimal and adapted protection devices and, at the same time, guarantee their durability.

During the impact of a block on a protection device, an energy exchange between the block and the structure occurs. The external work related to the forces applied onto the block at a given time $t\left(W_{E x t}\right)$ is equal to the block's kinetic energy variation from the impact beginning $\left(\Delta E_{k}^{b}=E_{k t}^{b}-E_{k i n}^{b}\right)$, where $E_{k i n}^{b}$ and $E_{k t}^{b}$ are the block's kinetic energy before impact and at time $t$, respectively.

$$
W_{E x t}=-\Delta E_{k}^{b}
$$

where $W_{E x t}$ is calculated from equation 12 . As regards to the protective structure, the energy balance can be expressed as follows for any time during impact:

$$
W_{E x t}=W_{\text {Int }}+W_{\text {Cont }}+\Delta E_{k}^{t}+W_{\text {Damp }}
$$

where $W_{\text {Int }}, W_{\text {Cont }}, \Delta E_{k}^{t}$, and $W_{\text {Damp }}$ are the stem's strain energy, the contact strain energy, the stem's kinetic energy variation from the impact beginning, and the energy dissipated by damping, respectively.

$W_{\text {Int }}$ is calculated as follows:

$$
d W_{\text {Int }}=\sum_{a=1}^{n-1} N_{a} \cdot d u_{a}+\sum_{a=1}^{n-1} T_{a} \cdot d v_{a}+\sum_{a=1}^{n-1} M_{a} \cdot d \theta_{z, a}
$$

where $a$ is the node interaction identifier and $n$ is the number of nodes. $N, T$ and $M$ are the normal and tangential components of the interaction force, and bending moment between nodes participating to the interaction $a . u_{a}$ and $v_{a}$ are the normal and tangential components of the relative displacements between nodes taking part to the interaction.

The stem kinetic energy variation $\left(\Delta E_{k}^{t}\right)$ is calculated from the nodes masses and velocities (translational and angular) (Olmedo et al., 2016).

The contact strain energy $W_{\text {Cont }}$ is assessed incrementally. The contact energy increment $d W_{\text {Cont }}$ is calculated as a function of the impact force $F$ and of the indentation increment $d \alpha$ : 


$$
d W_{\text {Cont }}=F . d \alpha
$$

The energy dissipated by damping $W_{\text {Damp }}$ is also assessed incrementally using the increment $d W_{\text {Damp }}$ calculated from $\theta$ and the damping contribution to the bending moment $\left(M_{\text {Damp }}\right)$.

$$
d W_{\text {Damp }}=M_{\text {Damp }} . d \theta
$$

\section{Results and discussion}

\subsection{Model calibration}

The results obtained from the model calibration are compared to those obtained from simulations which do not consider explicitly the block-stem interaction (Olmedo et al., 2016), and to the experimental data. Figure 5 depicts the time evolutions of the external work (Eq.12) for a typical situation. The integration of the contact model allows a better description of the experimental observations, especially at the early beginning of the contact and around the maximum mid-span displacement.

Similar values of the parameters $M O E, \sigma_{\text {Elast }}$, and $\sigma_{\text {Rupt }}$ are obtained for model calibrations that explicitly integrate or not block-stem contact (Tab.2).

The explicit modeling of the block-stem contact leads to reduce the damping parameter $\beta$ (Tab.2). Indeed, the contact model tends to limit vibrations with high frequencies. Thus, if the stem/block contact is not explicitly taken into account, high frequency vibrations have to be damped with larger values of $\beta$ parameter.

Differences between calibration values of $E_{N L}$ and $E_{U n l d}$ are also observed. $E_{N L}$ and $E_{U n l d}$ are the leading parameters for the modelling of correct values of the maximum stem deflection and contact force. And, near the maximum stem deflection, different structural responses occur if the block-stem contact is explictly modelled or not.

\subsection{Impact scenarii}

\subsubsection{Impact classification}

3.2.1.1. Impact duration $\left(t_{c}\right) . t_{c}$ is defined from the instant when the block-stem contact is established to the end of the last contact. The evolution of $t_{c}$ as a function of the ratio $R_{D}\left(R_{D}=\frac{D_{b}}{D_{t}}\right.$ - Figure 6$)$ shows that low diameter ratios $\left(R_{D}<1.5\right)$ are associated with short contact durations $\left(t_{c}<30 \mathrm{~ms}\right)$. In these cases, $t_{c}$ presents a small variability (from $10 \mathrm{~ms}$ to $30 \mathrm{~ms}$ ). For $R_{D}$ values between 1.2 and $2, t_{c}$ presents a larger variability, ranging from $70 \mathrm{~ms}$ to $150 \mathrm{~ms}$, with no clear dependency to $R_{D}$. Finally, for $R_{D}>2$ and $t_{c}>150 \mathrm{~ms}$, a linear increase of $t_{c}$ with $R_{D}$ is observed. This analysis tends to show a potential classification of the simulations in three groups (A, $\mathrm{B}$, and $\mathrm{C}$ ) presented in Figure 6.

The time evolutions of the impact forces between the block and the stem are depicted in Figure 7 for groups A, B, and C. For the group A, the block-stem interaction presents only one contact. For the group B, successive contacts (2 or 3 ) of similar durations can be identified. $t_{c}$ values are significantly higher for simulations presenting three contact 
phases, even if the latest is not significant in terms of force magnitude. Finally, group C simulations always present two contacts, the second being substantially longer than the first one. These clear differences in terms of time evolutions of the impact forces between the groups lead to classify each impact simulation.

3.2.1.2. Momentum variation $(\Delta p)$. The block's momentum variation $\Delta p$ $\left(\Delta p=m_{b}\left(v_{\text {in }}-v_{\text {out }}\right)\right.$, with $v_{\text {out }}$ the block's velocity after impact) is calculated to evaluate the variation in the block kinematics. The relation between the block's momentum variation $\Delta p$ and the diameter ratio $R_{D}$ is analysed. The normalized value $\overline{\Delta p}=\frac{\Delta p}{p_{i n}}$ is defined, where $p_{i n}$ is the block momentum just before the impact $\left(p_{i n}=m_{b} v_{i n}\right)$.

The $\overline{\Delta p}$ values for all simulations range between two particular cases. The first one is an elastic impact implying that $v_{\text {out }}$ is opposite to the incoming velocity $\left(v_{\text {out }}=\right.$ $\left.-v_{\text {in }}\right)$. In the second one, the block is stopped $\left(v_{\text {out }}=0\right)$. For the first case, $\overline{\Delta p}=2$ whereas, for the second one, $\overline{\Delta p}=1$. Thus, the lower $\overline{\Delta p}$, the larger the loss of block's momentum.

Figure 8 underlines that the groups $\mathrm{A}, \mathrm{B}$ and $\mathrm{C}$ present different trends in the evolution of $\overline{\Delta p}$ with $R_{D}$. Group A results present a linear decrease in $\overline{\Delta p}$ with increasing $R_{D}$ values. Group $\mathrm{B}$ results globally present higher $\overline{\Delta p}$ values and larger variability than group $\mathrm{A}$ data for a given $R_{D}$ value. Group $\mathrm{C}$ results, observed for large $R_{D}$, exhibit similar values as for group $\mathrm{B}$ with a slightly smaller variability.

The $R_{D}$ values delimiting the groups $\mathrm{A}, \mathrm{B}$, and $\mathrm{C}$ depend on the impact velocity (Figure 9): for impacts at $v_{i n}=5 \mathrm{~m} . \mathrm{s}^{-1}$ (resp. $\left.v_{i n}=30 \mathrm{~m} . \mathrm{s}^{-1}\right)$, the transition from group $\mathrm{A}$ to $\mathrm{B}$ is observed at $R_{D}^{A B} \simeq 1.5$ (resp. $R_{D}^{A B} \simeq 1.2$ ). The transition from group $\mathrm{B}$ to $\mathrm{C}$ appears at $R_{D}^{B C} \simeq 2$. (resp. $R_{D}^{B C} \simeq 1.55$ ) for impacts at $v_{\text {in }}=5 \mathrm{~m} . \mathrm{s}^{-1}$ (resp. $\left.v_{\text {in }}=30 \mathrm{~m} \cdot \mathrm{s}^{-1}\right)$.

3.2.1.3. Structure damage. The maximum bending moment $M_{M a x}$ achieved during each impact simulation is compared with $M_{\text {Elast }}$ and $M_{\text {Rupt }}$ for each simulation, allowing the assessment of stem damage for each case. If $M_{\text {Max }}<M_{\text {Elast }}$, no material non-linearities developed and no structure damage occurred. When $M_{\text {Elast }}<M_{\text {Max }}<$ $M_{\text {Rupt }}$, material non-linearities appeared but the structure did not break. Finally, if $M_{\text {Max }}>M_{\text {Rupt }}$, the rupture of the structure occurred. These three scenarii are called, zone 1,2 , and 3 .

Stem rupture (zone 3) is observed for groups B and C, specially for high impact velocities. On the contrary, for most of group A simulations, the struture is not damaged at all (zone 1), only few high speed (i.e. $v_{\text {in }}>20 \mathrm{~m}_{\mathrm{s}} \mathrm{s}^{-1}$ ) impacts present material non-linearities (zone 2) (Figure 10).

\subsubsection{Relative displacements between the block and the stem}

Three impact configurations representative of each response group are analysed to highlight the differences in terms of relative displacements between the block and the stem. The input parameters for each example case are summarized in Table 3. They correspond to impacts at $5 \mathrm{~m} \cdot \mathrm{s}^{-1}$.

The time evolutions of the block center of mass $\left(y_{b}\right)$ and the stem's mid-span $\left(y_{t}\right)$ displacements and velocities are presented in Figure 11. Block and stem are in contact when $\alpha=y_{b}-y_{t} \geqslant 0$ (c.f. red surfaces in Figure 11. Specific attention is paid to the block and stem velocities just after the first contact and at the beginning of the second contact. 
As observed previously, group A impacts present only one and short contact (Figure $11 \mathrm{a}, \mathrm{d})$. At the end of this short contact, the block velocity is negative. This value increases toward positive values for increasing $R_{D}$. Thus, the block is sent back more and more slowly for increasing $R_{D}$ which favors the occurrence of a second contact (Figure 11b, e). Group B impacts are observed if the block velocity after the first contact is positive. In addition, the second contacts occur for negative values of the stem velocity, i.e. during the stem way back from its initial oscillation.

For Group $\mathrm{C}$ impacts, i.e. for larger $R_{D}$ values, the block velocity after the first contact is positive and keeps increasing for increasing $R_{D}$ values (Figure 11f). Thus, the second contact occurs earlier in the stem oscillation process. It takes place for positive stem mid-span velocities. In these configurations, the block and the stem velocities directions are similar after the first contact. In consequence, the stem's midspan and the block displacements follow analogous evolutions after the first impact (Figure 11c) and till the end of the impact.

\subsubsection{Energy exchanges}

The energy balance during impact is analysed for the three cases representatives of each group.

For group A, the block's energy is nil at the end of the initial contact loading stage (i.e. $t \simeq 6 \mathrm{~ms}$ ) and most of the energy is transferred into energy at the contact $\left(W_{\text {Cont }}\right)$. The contact energy is transferred back from the contact to the block and the stem during the unloading phase of the initial contact (Figure 12a). As the interaction between the block and the stem is limited to the initial contact, the block's energy loss is mainly due to momentum transfer during the first contact and, possibly, to energy dissipation at the contact (up to $20 \%$ of the impact energy in some cases).

For group B, energy transfers during the first contact are very similar to those observed for the group A (Figure 12b). The momentum transfer during the first impact induces that the block energy is almost nil at the end of the first contact (i.e. $t \simeq 20$ $m s)$. On the contrary, the stem energy is significant between the first and second contact. It is periodically converted from kinetic to strain energy following the stem oscillation and substantial dissipation of energy occurs due to damping between the two contacts. During the second contact (i.e. $70 \mathrm{~ms} \lesssim t \lesssim 90 \mathrm{~ms}$ ), a significant amount of energy is transferred back from the stem to the block. When the second contact takes place, the stem kinetic energy is high and decreases to almost zero during the second contact. Energy dissipation may occur at the contact during the first contact, but not significantly during the second one.

For the group C, as for the groups A and B, the first contact leads to a significant momentum transfer. Similarly to group B, given the high stem kinetic energy after the first contact, the energy dissipation by damping between the two contacts is significant (between $10 \%$ and $20 \%$ of the impact energy). But, contrary to group B, when the second contact occurs, the stem strain energy is high and its kinetic energy is low (Figure 12c). Thus, the energy exchange during the second contact is driven by a quasi-static interaction between the stem and the block.

The block energy loss is due, for Group A, to a significant momentum transfer and to energy dissipation at the contact during the first contact. The occurence of a second contact leads to a second energy transfer from the stem to the block for groups B and C. For these groups, a significant energy amount is also dissipated by damping of the stem motion between the two contacts. For group B, the second contact induces a momentum exchange from the stem to the block because the stem kinetic energy 
is large and the block one is small at the beginning of the second contact. On the contrary for group C, the stem strain energy is large at the beginning of the second contact and, thus, the second contact can be assimilated to a quasi-static interaction.

\subsection{Towards design recommendations}

The validity of the model is limited to the impact conditions of the study in terms of block and stem properties as well as loading type, i.e. normal impact of a block far from the boundary of the structure, and structure size, in particular. As the model was not calibrated directly from real scale impact experiments, it seems more reasonable to use the results for a qualitative analysis rather than for quantative assessments. Despite these limitations, relevant information is provided for the design of wooden protection structures.

The design of rockfall protection structures is generally composed of two steps related to the structural and functional design. The functional design is dedicated to assess the capacity of the structure to stop the blocks and the structural design aims at quantifying the capacity of the structure to sustain the loading by the block (Lambert, \& Bourrier, 2013).

The functional capacity of the structure relies on the analysis of the block momentum reduction while the structural design is based on the study of structure damage. Concerning block momentum reduction, the choice was made to consider the stopping of the rock $(\overline{\Delta p}=1)$ as a target to assess the functional efficacy of the structure. On the contrary, a substantial velocity $(\overline{\Delta p}=2)$ is considered as the worst case in terms of functional efficacy among the configurations observed. For the structure damage, the optimal design corresponds to no damage of the structure.

A design approach can be proposed with the objective of favoring one or several of the three impact groups identified. Both the functional and structural efficacies of the structure can be estimated for the different groups. For group A, the block momentum reduction depends on a single and brief interaction. The momentum reduction increases for increasing diameter ratio to reach a maximum value among all groups observed for $R_{D}$ around 1.3. In addition, almost no structure damage is observed. On the contary, the momentum reduction is substantially larger for both group B and $\mathrm{C}$ while significant structure damage may occur. Thus, Group A impacts correspond to the target configuration both in terms of functional and structural designs.

In practice, as the block volume, i.e. diameter, is given for a study site, designing the structure to reach the target configuration consists in setting the tree diameter of the structure to obtain the target range of $R_{D}$. The analysis of the simulations allowed identifying optimal $R_{D}$ values. However, the results also show that the transition from an optimal configuration (group A) to an unfavorable one can occur for small variations of $R_{D}$. The choice of the tree diameter should thus be made with caution.

Finally, the analysis of the physical processes shows that the optimal configuration corresponds to a single and brief interaction between the block and the structure. Technical solutions can be prescribed to favor the occurrence of a single impact, i.e. to avoid the occurrence of a second momentum exchange leading to a group B impact. For this purpose, changes in the boundary conditions of the structure, such as fixing the structure to loose cables anchored on stumps instead of placing it directly on the stump, can be envisaged for example. 


\section{Conclusions and perspectives}

The DEM model developed integrates the description of the physical processes both at the stem and contact scales. After a calibration phase, the combined model, accounting for the contact between the block and the stem, has been used for real scale simulations.

Three types of impact have been identified depending on the ratio $R_{D}$ between the block radius and the stem diameter. A unique and brief contact occurs for small $R_{D}$ values. For intermediate $R_{D}$ values, at least two successive contacts of similar duration occur. The block continues in the same direction after the first contact and is sent back with a large velocity after the second one. For large $R_{D}$ values, two contacts are also observed. During the second contact, longer than the first one, a quasi-static loading of the stem occurs. Structure damage increases for increasing $R_{D}$ values : the first impact type entails almost no damage whereas substantial damage occur for the third type.

The optimal configuration in terms of structure design is a unique and brief contact between the block and the structure with a substantial decrease in the block momentum and almost no structure damage. This configuration corresponds to the largest $R_{D}$ values associated with the first impact type.

The model proposed is a computationally efficient tool usable for the study of the efficacy of wooden protection structures for a wide range of impact conditions and structure configurations. Further model developments are foreseen to simulate noncentered and oblique impacts and to integrate the interaction of the structure with the ground that may represent a major source of energy dissipation.

In addition, the relevance of the calibration of the model, done using experimental results on smalll diameters stems $\left(D_{t}<10 \mathrm{~cm}\right)$, for large tree diameters has been assessed based on comparisons to previous studies found in the literature only. This aspect of the development of the model could be improved by detailed studies of the mechanical properties of large diameter trees. These studies could be based on experimental studies found in the literature and dedicated to the assesment of the mechanical properties of trees at the real-scale (Ruel et al., 2010; Schonenberger et al., 2005) or to field experiments carried out at the tree scale (Lundstrom et al., 2007).

Finally, a major improvement of the model would consist in the integration of an appropriate mechanical description of decayed wood into the model to estimate the time evolution of the protective capacity of these structures. This improvement would concern both the model of the stem mechanical properties and of the structure boundaries. Indeed, both of these components of the wooden structures are affected by wood degradation (Benfratello et al., 2017; Romano et al., 2016). For that purpose, detailled studies have to be carried out to characterize the time evolution of the mechanical properties of the stems and of the structure boundaries due to degradation processes (i.e. fungi and entomofaune aggression in particular).

\section{Acknowledgements}

This work was supported by the Natural Hazards and Vulnerability of Structures (VOR) research network. This network is funded by the French Ministry of Research and joins different laboratories in the Rhônes-Alpes region. 


\section{References}

Abrate, S. (1998). Impact on composite structures. Cambridge Univ. Press.

Benfratello, S., Di Stefano, C., Ferro, V., and Palizzolo, L. (2017). Testing mechanical characteristics of chestnut stakes used in bed sills for stream restoration. Journal of Agricultural Engineering, 480(3), 161-166.

Bergman, R., Cai, Z., and Carll, C. (2010). Wood handbook - Wood as engineering material. United States Department of Agriculture, Madison, WI.

Bertrand, D., Trad, A., Limam, A., and Silvani, C. (2012). Full-scale dynamic analysis of an innovative rockfall fence under impact using the discrete element method: from the local scale to the structure scale. Rock Mechanics and Rock Engineering, 45, 885-900.

Bertrand, D., Bourrier, F., Olmedo, I., Brun, M., Berger, F., and Limam, A. (2013). Experimental and numerical dynamic analysis of a live tree stem impacted by a Charpy pendulum. International Journal of Solids and Structures, 50, 1689-1698.

Bodig, J. and Jayne, B. (1982). Mechanics of wood and composites. Van Nostrand Reinhold.

Bourrier, F., Kneib, F., Chareyre, B., and Fourcaud, T. (2013). Discrete modeling of granular soils reinforcement by plant roots. Ecological Engineering, 61, 646-657.

Cazzani, A., Mongiovi, L., and Frenez, T. (2002). Dynamic finite element analysis of interceptive devices for falling rocks. International Journal of Rock Mechanics and Mining Sciences, 39, 303-321.

Crook, A. W. (1952). A Study of Some Impacts between Metal Bodies by a Piezo-Electric Method. Proceedings of the Royal Society of London A: Mathematical, Physical and Engineering Sciences, 212, 377-390.

Cundall, P. A., and Strack, O. D. L. (1979) A discrete numerical model for granular assemblies. Geotechnique 29(1), 47-65.

Dorren, L., Berger, F., Le Hir, C., Mermin, E., and Tardif, P. (2005). Mechanisms, effects and management implications of rockfall in forests. Forest Ecology and Management, 215, 183-195.

Dorren, L., Berger, F., and Putters, U. (2006). Real-size experiments and 3-D simulation of rockfall on forested and non-forested slopes. Natural Hazards and Earth System Sciences, $6,145-153$.

Effeindzourou, A., Chareyre, B., Thoeni, K., Giacomini, A., and Kneib, F. (2016) Modelling of deformable structures in the general framework of the discrete element method. Geotextiles and Geomembranes 44(2), 143-156.

Gottardi, G. and Govoni, L. (2009). Full-scale modelling of falling rock protection barriers. Rock Mechanics and Rock Engineering, 43, 261-274.

IGN, i. n. d. l. g. e. f. (2014). Inventaire forestier national franais: Les rsultats des campagnes dinventaire 2009 2013. Ministre de l'agriculture, de l'agroalimentaire et de la fort.

Jonsson, M. J. (2007). Energy absorption of trees in a rockfall protection forest. PhD thesis. Swiss Federal Institute of Technology, Zurich.

Keith, C.T. (1964). Annual layers effect resistance of wood to impact. Forest Products Journal, 14(7), 285-289.

Lambert, S. and Bourrier, F. (2013). Design of rockfall protection embankments: a review. Engineering Geology, 154, 77-88.

Lavers, G. and Gwendoline, M. (1974). The strength properties of timber. MTP Construction.

Lundstrom, T., Heiz, U., Stoffel, M., and Stockli, V. (2007). Fresh-wood bending: linking the mechanical growth properties of a Norway spruce stem. Tree Physiology, 27, 1229-1241.

Lundstrom, T., Jonsson, M., and Kalberer, M. (2007). The root-soil system of Norway spruce 
subjected to turning moment: resistance as a function of rotation. Plant Soil, 300(1-2), 35-49.

Lundstrom, T., Jonas, T., and Volkwein, A. (2008). Analysing the mechanical performance and growth adaptation of Norway spruce using a non-linear finite-element model and experimental data. Journal of Experimental Botany, 59(9), 2513-2528.

Olmedo, I., Bourrier, F., Bertrand, D., Toe, D., Berger, F., and Limam, A. (2015). Experimental analysis of the response of fresh wood stems subjected to localized impact loading. Wood Science and Technology, 49, 623-646.

Olmedo, I., Bourrier, F., Bertrand, D., Berger, F., and Limam, A. (2016). Discrete element model of fresh wood's dynamic response to impact. Engineering Structures, 120, 13-22.

O'Sullivan, C. (2012). Particle-based discrete element modeling: Geomechanics perspective. International Journal of Geomechanics, 11, 448-464.

Plassiard, J. and Donze, F. (2009). Rockfall impact parameters on embankments: a discreteelement method analysis. Structural Engineering International, 19, 333-341.

Quetel, C. (2005). Approche multi-chelle du comportement mcanique d'un arbre soumis l'impact d'un bloc rocheux (Multi-scale approach of tree response to a rockfall impact). Ph.D. thesis, Ecole Centrale de Lyon.

Romano, N., Lignola, G.P., Brigante, M., Bosso, L., and Chirico G.B. (2016). Residual life and degradation assessment of wood elements used in soil bioengineering structures for slope protection. Ecological Engineering, 90, 498-509.

Ronco, C., Oggeri, C., and Peila, D. (2009). Design of reinforced ground embankments used for rockfall protection. Natural Hazards and Earth System Sciences, 9, 1189-1199.

Ruel, J.C., Achim, A., Herrera, R.E., and Cloutier, A. (2010). Relating mechanical strength at the stem level to values obtained from defect-free wood samples. Trees, 24, 1127-1135.

Schonenberger, W., Noack, A., Thee, P. (2005). Effect of timber removal from windthrow slopes on the risk of snow avalanches and rockfall. Forest Ecology and Management, 213, 197-208.

Smilauer, V. and Chareyre, B. (2010) Yade documentation. The Yade project. http://yadedem.org/doc/.

Volkwein, A., Roth, A., Gerber, W., and Vogel, A. (2009). Flexible rockfall barriers subjected to extreme loads. Structural Engineering International, 19, 327-332.

Volkwein, A., Schellenberg, K., Labiouse, V., Agliardi, F., Berger, F., Bourrier, F., Dorren, L.K.A., Gerber, W., and Jaboyedoff, M. (2011) Rockfall characterisation and structural protection - A review. Natural Hazards and Earth System Science 11(9), 2617-2651 


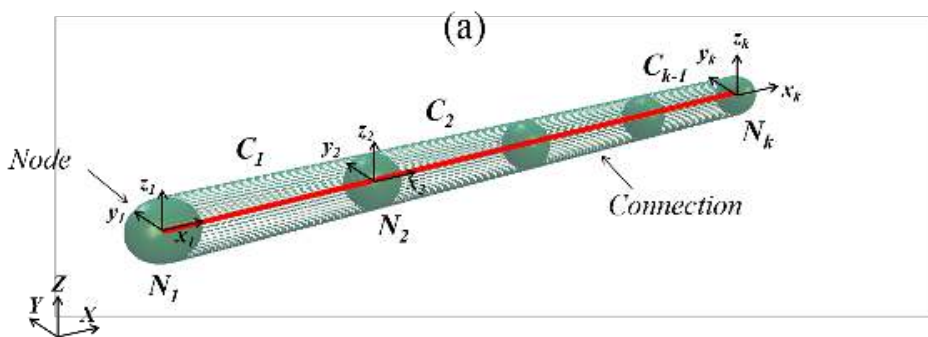

(b)

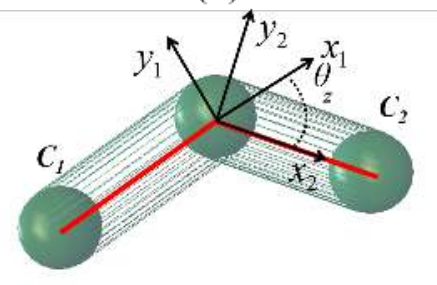

Figure 1.: Yade-DEM elements are composed of nodes and connections (a). The forces and moments applied to contiguous nodes are calculated from their relative positions and orientations (b). 


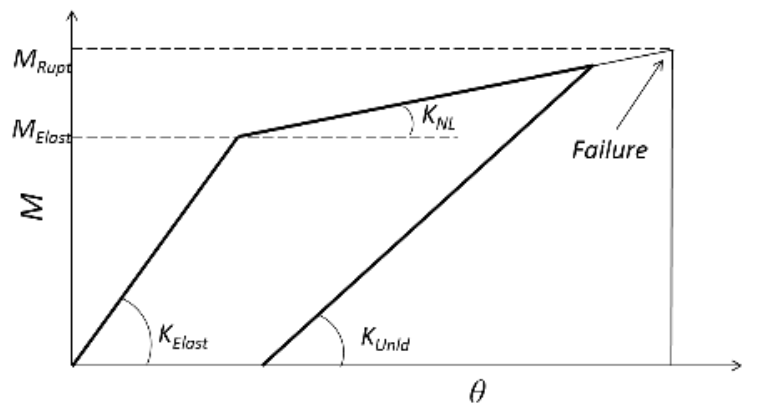

Figure 2.: Relation for the calculation of bending moments between contiguous nodes. 


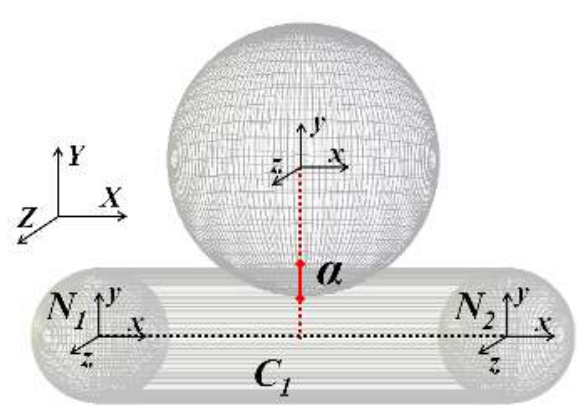

Figure 3.: Block-stem contact : the indentation $\alpha$ is calculated from the overlap between the bodies. 


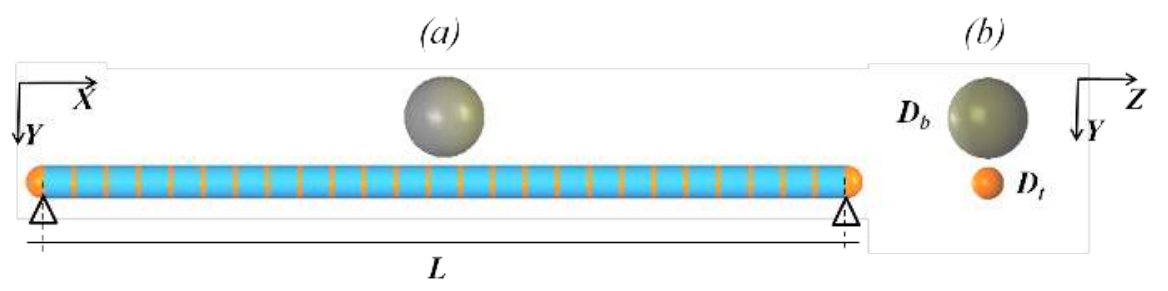

Figure 4.: DEM model accounting for the block-stem interaction. Face (a) and side (b) views. 


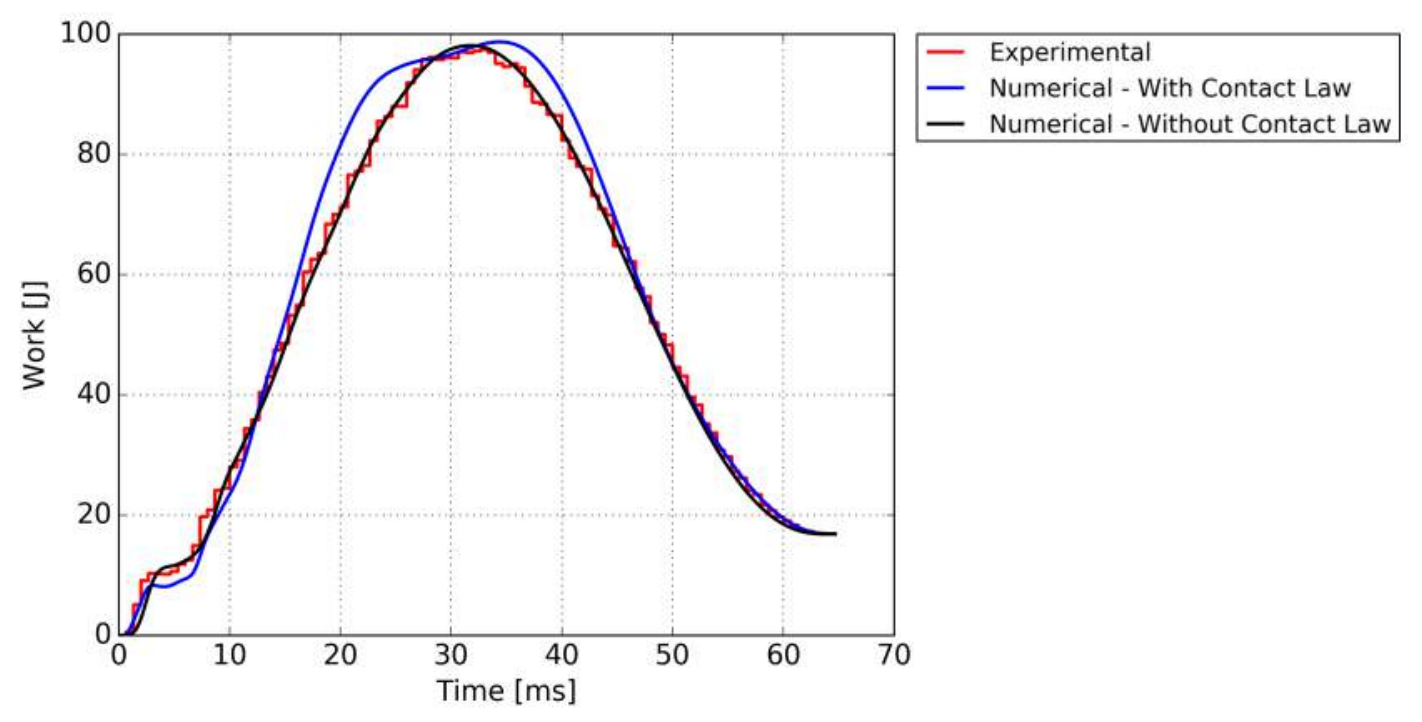

Figure 5.: Time evolutions of the external works for the experimental results (red) and numerical predictions with imposed loading force on the stem (blue) and explicit block-stem interaction (black) for a typical impact test. 


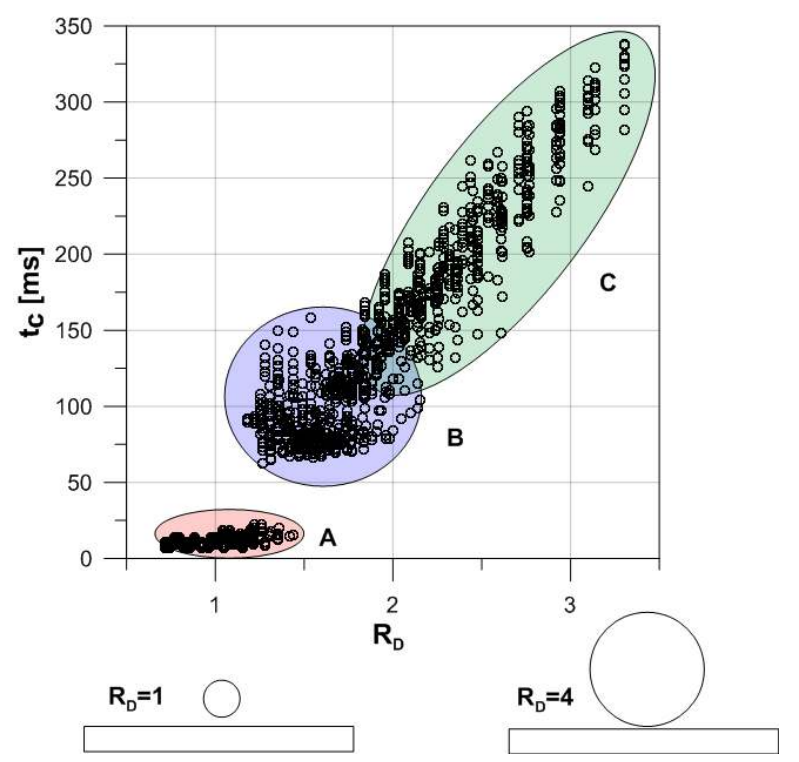

Figure 6.: Contact durations $\left(t_{c}\right)$ vs. diameter ratio $\left(R_{D}=D_{b} / D_{t}\right)$. Identification of the groups A (red), B (blue), and C (green). 


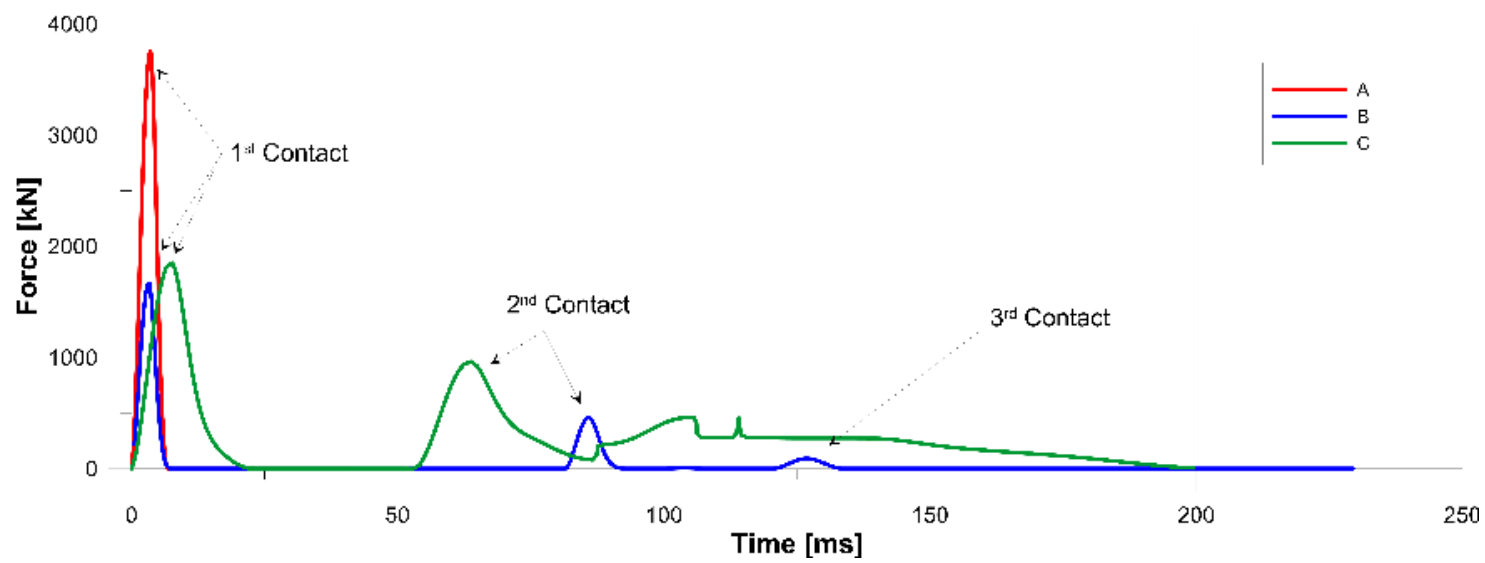

Figure 7.: Time evolutions of the contact force for three typical simulations belonging to the groups A (red), B (blue) and C (green). 


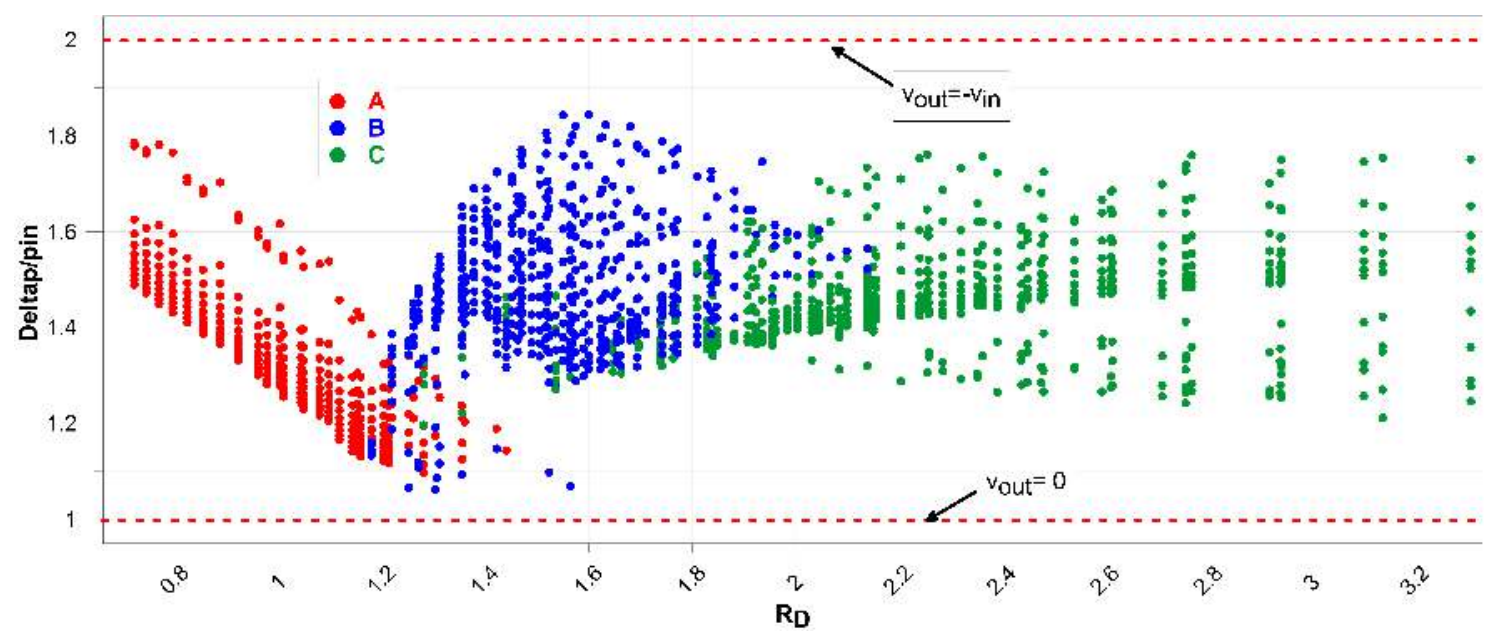

Figure 8.: Normalized momentum variation $(\overline{\Delta p})$ vs. $R_{D}$. The groups are depicted in red $(\mathrm{A})$, blue $(\mathrm{B})$ and green $(\mathrm{C})$. The dashed lines show the cases of a block stopped $\left(v_{\text {out }}=0\right)$ and of no block energy loss $\left(v_{\text {out }}=-v_{\text {in }}\right)$. 


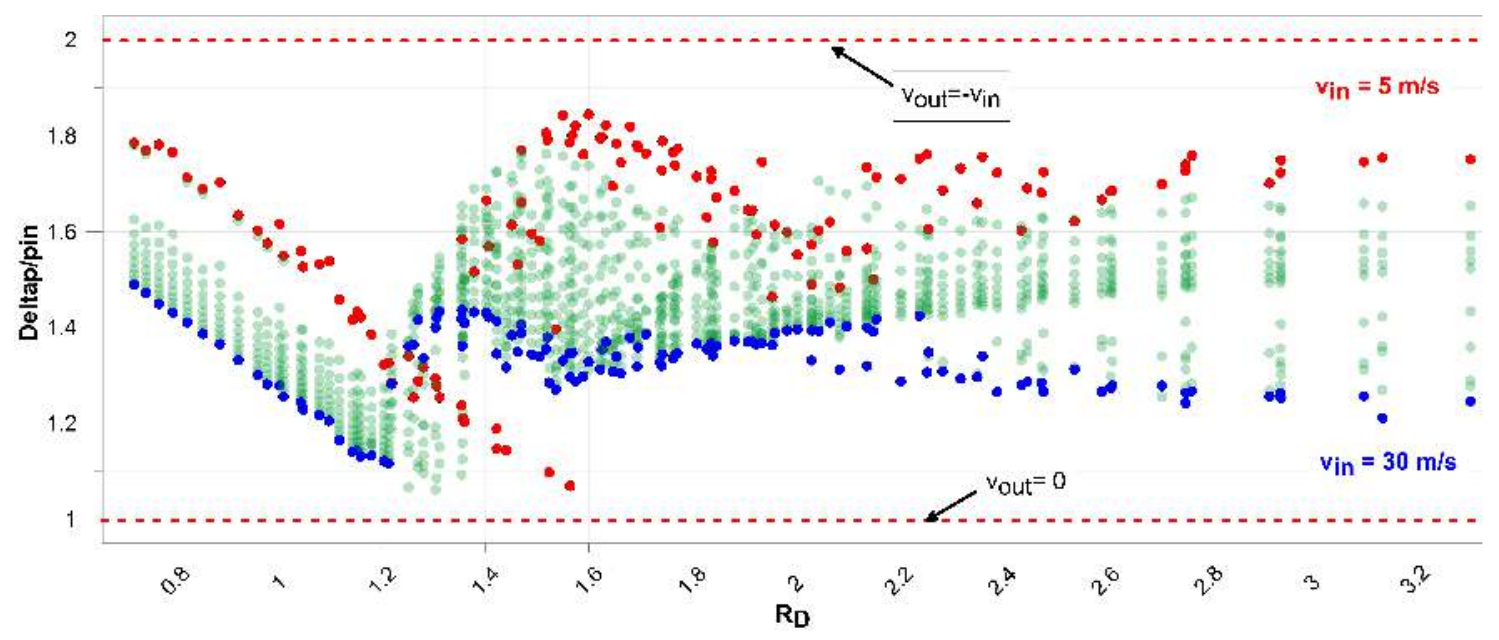

Figure 9.: Evolution of $\overline{\Delta p}$ with $R_{D}$ emphasizing impact velocities of $5 \mathrm{m.s} \mathrm{s}^{-1}$ (red) and $30 \mathrm{~m} \cdot \mathrm{s}^{-1}$ (blue). 


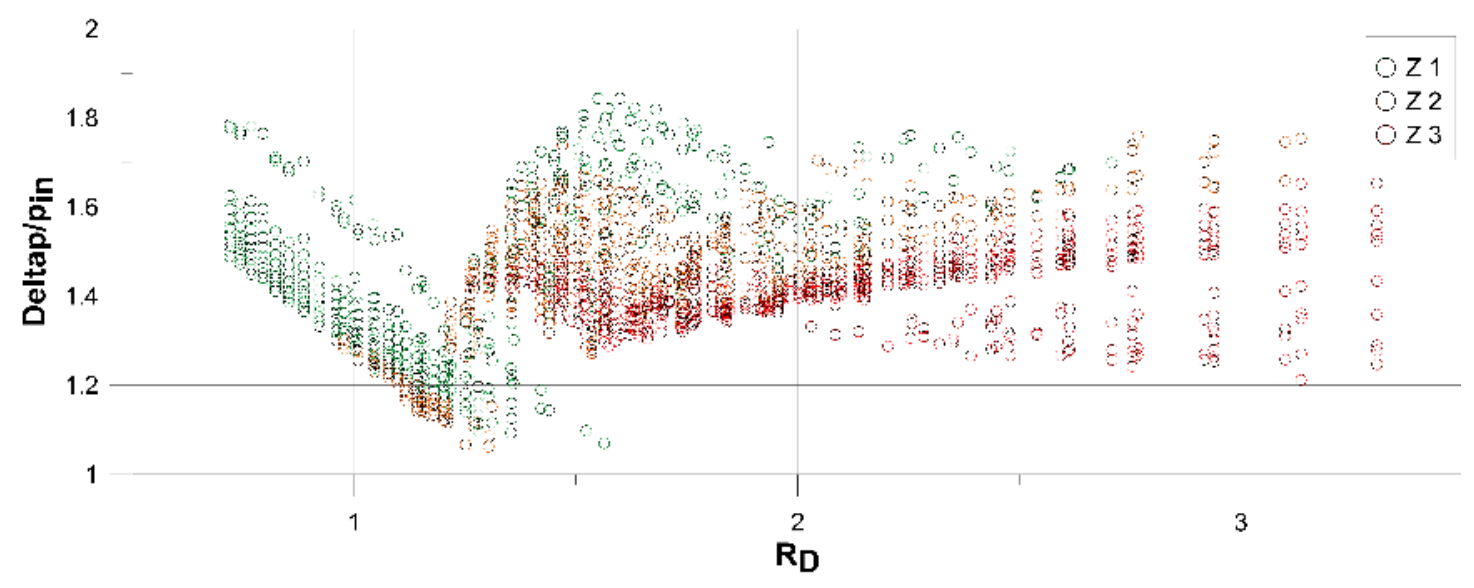

Figure 10.: Evolution of $\overline{\Delta p}$ with $R_{D}$ emphasizing the elastic $(z 1)$, non-linearities occurrence $(z 2)$ and rupture $(z 3)$ zones. 

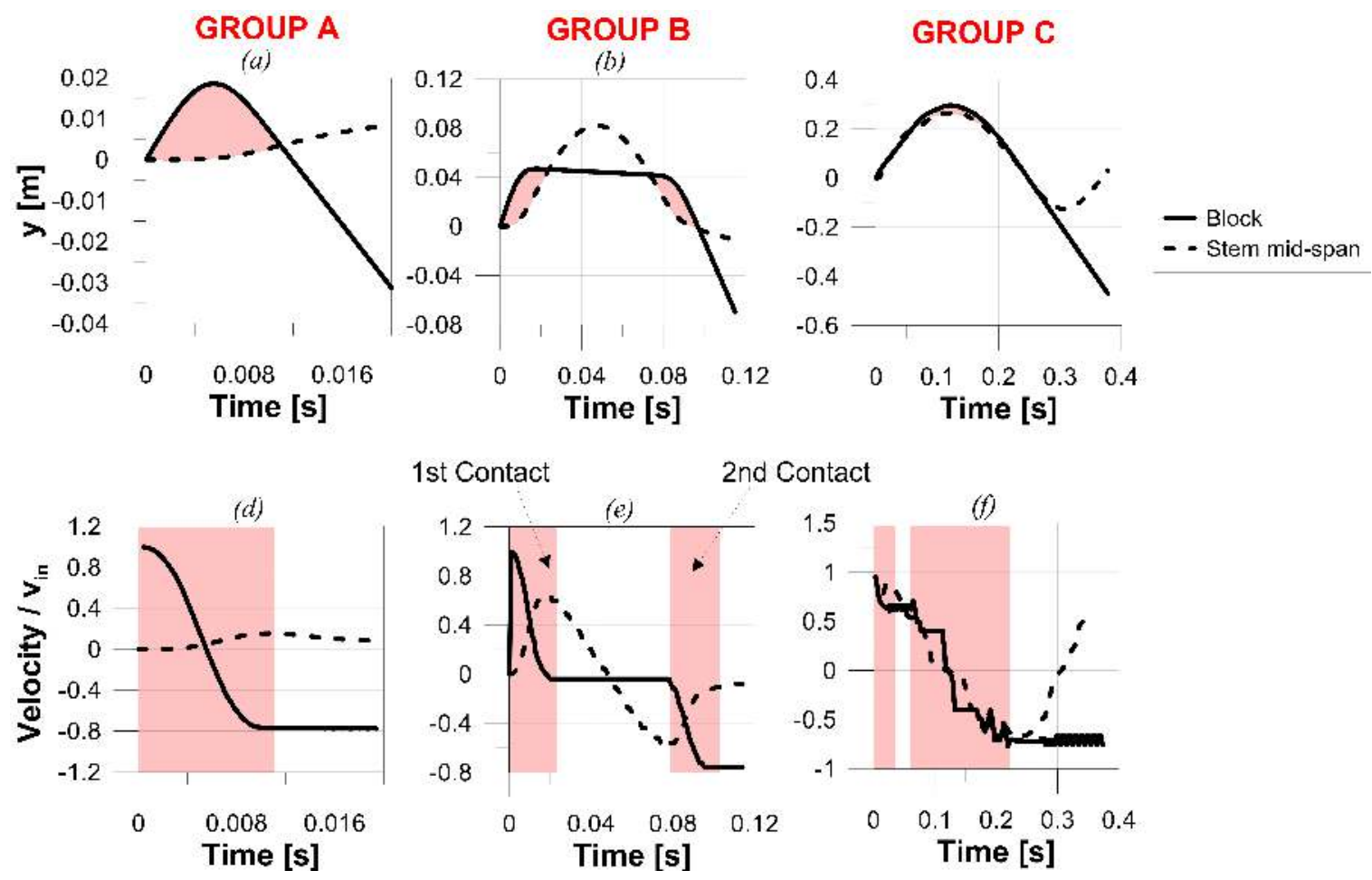

Figure 11.: Top: time evolutions of the mid-span (dashed line) and block (solid) displacements for group A (a), B (b) and C (c) simulations ; Bottom: time evolutions of the stem's mid-spam (dashed line) and block's (solid) velocities normalized by the impact velocity $v_{i n}$ for group A (d), B (e) and C (f) simulations. Red surfaces indicate the block-stem contacts $(\alpha>0)$. 


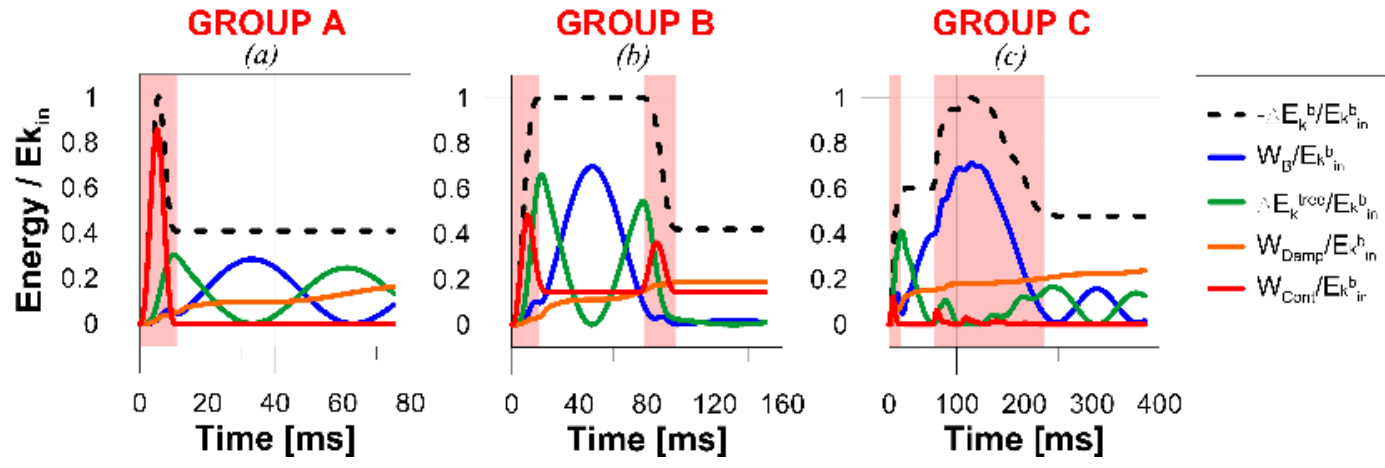

Figure 12:: Time evolutions of block and stem energies normalized by $E k_{\text {in }}^{b}$ : kinetic $\left(-\Delta E_{k}^{b}\right.$ and $\left.-\Delta E_{k}^{t}\right)$, bending strain $\left(W_{B}\right)$, damping dissipation $\left(W_{D a m p}\right)$ and contact $\left(W_{\text {Cont }}\right)$ energies. Shear and normal strain energies are negligible and, thus, not represented on the graphs. 


\begin{tabular}{ccc} 
& Min. & Max. \\
\hline$v_{\text {in }}\left[\mathrm{m.s} \mathrm{s}^{-1}\right]$ & 5 & 30 \\
\hline$D_{b}-V_{b}\left[\mathrm{~cm}-\mathrm{m}^{3}\right]$ & $57-0.1$ & $124-1$ \\
\hline$D_{t}[\mathrm{~cm}]$ & 20 & 80
\end{tabular}

Table 1.: Input values ranges considered for the real scenarii simulations. 


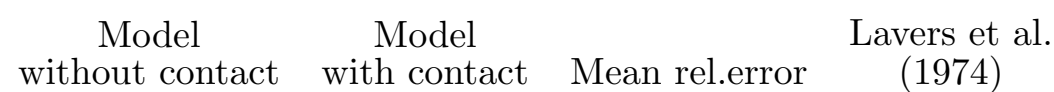

\begin{tabular}{ccccc}
\hline MOE $[\mathrm{MPa}]$ & 8940 & 9200 & $2.9 \%$ & 9800 \\
\hline$E_{N L}[\mathrm{MPa}]$ & 2070 & 2377 & $14.8 \%$ & - \\
\hline$E_{\text {Unld }}[\mathrm{MPa}]$ & 8820 & 6984 & $-20.7 \%$ & - \\
\hline$\sigma_{\text {Elast }}[\mathrm{MPa}]$ & 44.2 & 46.1 & $4.3 \%$ & - \\
\hline$\sigma_{\text {Rupt }}[\mathrm{MPa}]$ & 72.7 & 72.7 & $0 \%$ & 65. \\
\hline$\beta$ & $4.6 \mathrm{E}-4$ & $4.1 \mathrm{E}-4$ & $-10.8 \%$ & -
\end{tabular}

Table 2.: Mean values of the calibration parameters with and without explicit modelling of the block-stem contact compared with the MOE and MOR values proposed by Lavers, \& Gwendoline (1974). The relative error $\left(\frac{c_{\text {combined }}-c_{R e s p}}{c_{R e s p}}, c\right.$ corresponds to the different calibration parameters) quantifies the difference between calibration parameters values. 


\begin{tabular}{cccc} 
& $A$ & $B$ & $C$ \\
\hline$D_{b}[\mathbf{c m}]$ & 57.5 & 91.5 & 117.5 \\
\hline$D_{t}[\mathbf{c m}]$ & 75. & 57.5 & 60. \\
\hline$R_{D}$ & 0.77 & 1.59 & 1.95
\end{tabular}

Table 3.: Simulations representative of the groups A, B and C. 